- Original Article

\title{
Effects of Age at First Childbirth and Other Factors on Central Obesity in Postmenopausal Women: The 2013- 2015 Korean National Health and Nutrition Examination Survey
}

\author{
Wang Jin Lee', Jung Won Yoon ${ }^{2, *}$, Joo Ha Lee', Byoung Gyu Kwag', Shin Hae Chang', Yu Jin Choi ${ }^{1}$ \\ ${ }^{1}$ Department of Family Medicine, Green Hospital, Seoul, Korea \\ ${ }^{2}$ Department of Obstetrics and Gynecology, Green Hospital, Seoul, Korea
}

Background: Waist circumference is one of the key components of metabolic syndrome. Recent studies demonstrated that the reproductive profile was associated with metabolic syndrome in postmenopausal women. This study focused on the association between central obesity and age at first childbirth. It also considered other factors associated with central obesity in postmenopausal women.

Methods: This study was based on the 2013-2015 Korean National Health and Nutrition Examination Survey and involved 3,143 naturally postmenopausal women. These women were divided into three groups according to their age at first childbirth: 19 years or younger $(n=252), 20-29$ years $(n=2,695)$, and 30 years or older $(n=196)$. Multivariate analysis using logistic regression was performed to evaluate the effects of various reproductive factors, including other confounding factors.

Results: During adjustment for confounding factors, in the early age at first childbirth group, odds ratios (95\% confidence intervals) for central obesity decreased. In the final model, younger age at first childbirth was not significantly related to central obesity (waist circumference more than $85 \mathrm{~cm}$ ) in naturally postmenopausal women after adjusting for other confounding factors.

Conclusion: Younger age at first childbirth was not significantly associated with central obesity after adjustment for confounding factors.

Keywords: Waist Circumference; Abdominal Obesity; Age; Parturition; Menopause

Received: December 12, 2016, Revised: April 24, 2017, Accepted: May 23, 2017

*Corresponding Author: Jung Won Yoon https://orcid.org/0000-0001-8648-746X

Tel: +82-2-490-2134, Fax: +82-2-490-2222, E-mail: erynies4@gmail.com 


\section{INTRODUCTION}

Pregnancy is a time-limited condition, but it can cause many physiological changes in women during the gestation period and later in life. Insulin resistance, thrombophilia, immunosuppression, and hypervolemia can occur during pregnancy, while cardiovascular and metabolic diseases can develop from preeclampsia and gestational diabetes later in life. ${ }^{1)}$ Teenage mothers have a higher risk of all-cause mortality, including cardiovascular mortality. ${ }^{2,3)}$ In addition, childbirth during adolescence is associated with a higher risk of diabetes-related mortality in postmenopausal women. ${ }^{4)}$ Furthermore, menopause can induce certain medical conditions in women, such as metabolic syndrome, osteoporosis, and cardiovascular disease. ${ }^{5)}$ In premenopausal women, reproductive hormones have protective effects on physiological and metabolic mechanisms; thus, the prevalence of metabolic syndrome increases with age because of the deficiency of reproductive hormones in postmenopausal women. Metabolic syndrome includes weight gain, high blood pressure (BP), insulin resistance, and dyslipidemia. ${ }^{6,7)}$ Central obesity is an essential criterion used to diagnose metabolic syndrome. ${ }^{7)}$ According to the waist circumference cutoff modifications for Korean women, the criterion for central obesity in adult women is defined as a waist circumference of more than $85 \mathrm{~cm}^{8}{ }^{8)}$ Central obesity is also associated with chronic diseases leading to death. ${ }^{9)}$ Therefore, the interest in investigating risk factors for obesity has increased. Fat redistribution and central obesity are strongly linked to female reproductive hormones. ${ }^{10)}$ However, there is little information regarding the definite cause of central obesity and metabolic syndrome. ${ }^{11)}$ In some cases, central obesity is related to socioeconomic status. Younger age at first childbirth is sometimes associated with a disruption in education and occupational attainment, which can increase the risk of obesity. ${ }^{12,13)}$ Therefore, we suggest that central obesity may be correlated with age at first childbirth. The purpose of this study was to assess the effects of age at first childbirth and other factors on central obesity in postmenopausal women.

\section{METHODS}

\section{Subjects}

This study was based on data from the Korean National Health and Nutrition Examination Survey conducted by the Korean Ministry of Health and Welfare from 2013 to 2015. Written informed consent was obtained from all survey participants. Of the 22,948 participants involved in the 2013-2015 survey, we used data from 3,143 postmenopausal women. We excluded those with missing or incomplete data. Menopause was limited to natural menopause, which was defined as the absence of menses for 12 consecutive months.

\section{Measurements}

Body mass index (BMI) was calculated as weight in kilograms divided by the square of height in meters. Waist circumference was measured at the midpoint between the lower margin of the last rib and the top of the iliac crest during end expiration. BP was measured in the right arm using a standard mercury sphygmomanometer after 5 minutes of rest in the sitting position. The mean value of two separate BP measurements was used for analysis. Elevated BP was defined as systolic $\geq 130$ $\mathrm{mm} \mathrm{Hg}$ and diastolic $\geq 85 \mathrm{~mm} \mathrm{Hg}$. Blood samples were obtained after more than 8 hours of fasting. Elevated triglyceride (TG $\geq 150 \mathrm{mg} / \mathrm{dL}$ ) levels, reduced high-density lipoprotein cholesterol (HDL cholesterol $<50 \mathrm{mg} / \mathrm{dL}$ for women), and elevated fasting plasma glucose (FPG $\geq 100 \mathrm{mg} / \mathrm{dL}$ ) levels were found. Smoking, alcohol consumption, and regular walking indicated on self-reported questionnaires were categorized as lifestyle factors. Alcohol consumption was divided into three groups: never, social $(\leq 2-4$ times per month and $\leq 4$ units per day), and heavy ( $\geq 2-3$ times per week or $\geq 5$ units per day). Regular walking was defined as walking for 30 minutes or more per day for 5 days or more per week. Self-reported family income and education were categorized as socioeconomic factors. Known hypertension, diabetes, and dyslipidemia were diagnosed by a physician. Data regarding age at menarche, age at menopause, number of pregnancies, breastfeeding, and oral contraceptive use were acquired by self-reported questionnaires and were categorized as reproductive factors, except for age at menopause. The reproductive period was used instead of using age at menopause as a reproductive factor. The reproductive period was defined as the time from the age at menarche to the age at menopause.

\section{Metabolic Syndrome}

We defined metabolic syndrome using the definition of the American Heart Association/National Heart, Lung, and Blood Institute. ${ }^{14)}$ The definition comprised the presence of three or more of the following components: large waist circumference ( $\geq 90 \mathrm{~cm}$ for men, $\geq 85 \mathrm{~cm}$ for women); ${ }^{8)} \mathrm{TG} \geq 150 \mathrm{mg} / \mathrm{dL}$, HDL cholesterol ( $<50 \mathrm{mg} / \mathrm{dL}$ for women and $<40 \mathrm{mg} / \mathrm{dL}$ for men), high BP ( $\geq 130 / 85 \mathrm{~mm} \mathrm{Hg}$ ) or treatment for hypertension, and fasting glucose level $\geq 100 \mathrm{mg} / \mathrm{dL}$ or treatment for diabetes.

\section{Statistical Analysis}

Continuous variables with skewed distributions were expressed as median and interquartile range; categorical variables were expressed as numbers and percentages. The Kruskal-Wallis test followed by posthoc analysis using the Bonferroni method was used for continuous variables with skewed distributions. A chi-square test for categorical variables was used to compare frequencies among the groups. To estimate differences related to age at first childbirth, women were divided into three groups (19 years or younger, 20-29 years, and 30 years or older at first childbirth). Central obesity was defined as a waist circumference of $85 \mathrm{~cm}$ or more. Multiple logistic regression analysis was used to evaluate the odds ratio (OR) for measuring the association between central obesity and age at first childbirth. To obtain a final model, the backward elimination method was used $(\mathrm{P}<0.05$ : selection; $\mathrm{P}>0.10$ : elimination). Confounding factors were adjusted in a series of models. Covariates were added to the model as follows: model 1 (un- 
adjusted), age at first childbirth; model 2 (adjusted), age was added to model 1; model 3 (adjusted), lifestyle factors (smoking, alcohol consumption, and regular walking) were added to model 2; model 4 (adjusted), socioeconomic factors (family income and education) were added to model 3; and model 5 (adjusted), reproductive factors (age at menarche, number of pregnancies, breastfeeding, oral contraceptive use, and reproductive period) were added to model 4 . All continuous values with skewed distributions, such as age, BMI, age at menarche, reproductive period, age at first childbirth, and number of pregnancies, were log-transformed prior to the analysis. Age at first childbirth of 20-29 years was defined as the reference. Age at first childbirth was 20-29 years for the majority of women (reference group) involved in this study. We could hypothesize that the reference group was at low risk for central obesity later in life because they did not have a first pregnancy during adolescence or at an advanced maternal age. ${ }^{13,15)}$ First childbirth for those 19 years or younger was defined as adolescent pregnancy. Advanced maternal age is generally defined as 35 years or older during pregnancy. ${ }^{16)}$ In this study, first childbirth at age 35 years or older was very rare. Therefore, we defined age 30 years at first childbirth as the cutoff age for this study. Statistical analysis was performed using IBM SPSS ver. 23.0 (IBM Corp., Armonk, NY, USA); $\mathrm{P}<0.05$ was considered statistically significant.

\section{RESULTS}

Table 1 presents the characteristics of the three groups by age at first childbirth. A total of 3,143 participants were included, comprising women who were 19 years or younger $(8.0 \%), 20-29$ years $(85.7 \%)$, and

Table 1. Characteristics of the study population according to age at first childbirth

\begin{tabular}{|c|c|c|c|c|}
\hline \multirow{2}{*}{ Characteristic } & \multicolumn{3}{|c|}{ Age (y) } & \multirow{2}{*}{ P-value } \\
\hline & $\leq 19(n=252)$ & $20-29(n=2,695)$ & $\geq 30(n=196)$ & \\
\hline Total no. & $252(8.0)$ & $2,695(85.7)$ & $196(6.2)$ & \\
\hline Age $(y)$ & $69(61-75)$ & $63(56-70)$ & $58(54-67)$ & $<0.001$ \\
\hline Body mass index $\left(\mathrm{kg} / \mathrm{m}^{2}\right)$ & $24.8(22.7-26.9)$ & $23.7(21.9-25.9)$ & $23.3(21.5-25.1)$ & $<0.001$ \\
\hline Central obesity & $131(52.0)$ & $938(34.8)$ & $54(27.6)$ & $<0.001$ \\
\hline Elevated blood pressure & $115(45.6)$ & $1,041(38.6)$ & $56(28.6)$ & 0.001 \\
\hline Elevated triglyceride & $89(35.3)$ & $788(29.2)$ & $48(24.5)$ & 0.038 \\
\hline Reduced high-density lipoprotein cholesterol & $133(52.8)$ & $1,340(49.7)$ & $90(45.9)$ & 0.354 \\
\hline Elevated fasting plasma glucose & $115(45.6)$ & $1,077(40.0)$ & $69(35.2)$ & 0.075 \\
\hline Smoking & & & & $<0.001$ \\
\hline Never & $209(82.9)$ & $2,568(95.3)$ & $180(91.8)$ & \\
\hline Past & $19(7.5)$ & $62(2.3)$ & $9(4.6)$ & \\
\hline Current & $24(9.5)$ & $65(2.4)$ & $7(3.6)$ & \\
\hline Alcohol consumption & & & & 0.151 \\
\hline Never & $133(52.8)$ & $1,339(49.7)$ & $102(52.0)$ & \\
\hline Social & $90(35.7)$ & $1,113(41.3)$ & $83(42.3)$ & \\
\hline Heavy & $29(11.5)$ & $243(9.0)$ & $11(5.6)$ & \\
\hline Regular walking & $42(16.7)$ & $469(17.4)$ & $34(17.3)$ & 0.957 \\
\hline Family income & & & & $<0.001$ \\
\hline Low & $127(50.4)$ & 778 (28.9) & $49(25.0)$ & \\
\hline Low-mid & $69(27.4)$ & $710(26.3)$ & $57(29.1)$ & \\
\hline Mid-high & $36(14.3)$ & $602(22.3)$ & $42(21.4)$ & \\
\hline High & $20(7.9)$ & $605(22.4)$ & $48(24.5)$ & \\
\hline Education & & & & $<0.001$ \\
\hline Elementary & $214(84.9)$ & $1,324(49.1)$ & $53(27.0)$ & \\
\hline Middle & $25(9.9)$ & $460(17.1)$ & $33(16.8)$ & \\
\hline High & $13(5.2)$ & $648(24.0)$ & $66(33.7)$ & \\
\hline$\geq$ College & 0 & $263(9.8)$ & $44(22.4)$ & \\
\hline Known hypertension & $137(55.4)$ & 995 (36.9) & $58(29.6)$ & $<0.001$ \\
\hline Known dyslipidemia & $69(27.4)$ & $805(29.9)$ & $45(23.0)$ & 0.097 \\
\hline Known diabetes & $48(19.0)$ & $329(12.2)$ & $22(11.2)$ & 0.006 \\
\hline Age at menarche $(\mathrm{y})$ & $15(14-16)$ & $15(14-17)$ & $15(14-16)$ & 0.018 \\
\hline Age at menopause $(\mathrm{y})$ & $50(46-53)$ & $50(48-53)$ & $50(48-53)$ & 0.195 \\
\hline Reproductive period (y) & $34(31-38)$ & $35(32-38)$ & $36(32-38)$ & 0.095 \\
\hline Age at first childbirth (y) & $19(18-19)$ & $24(22-26)$ & $32(31-34)$ & $<0.001$ \\
\hline No. of pregnancies & $5(4-7)$ & $4(3-6)$ & $3(2-4)$ & $<0.001$ \\
\hline Breastfeeding, ever & $248(98.4)$ & $2,460(91.3)$ & $140(71.4)$ & $<0.001$ \\
\hline Oral contraceptive, ever used & $82(32.5)$ & $593(22.0)$ & $25(12.8)$ & $<0.001$ \\
\hline
\end{tabular}

Values are presented as number (\%) for categorical variables and median (interquartile range) for continuous variables.

${ }^{*}$ Obtained using Kruskal-Wallis test and Pearson's chi-square test. 
30 years or older (6.2\%). Groups with younger age at first childbirth had higher median values for age, BMI, and number of pregnancies; these values showed significant differences between the three groups. Significant differences were found for age at menarche between those aged 20-29 years and those aged 30 years or older at first childbirth during the post-hoc analysis. However, no significant differences were found for age at menopause or reproductive period for the three groups. The percentages of central obesity, elevated BP, elevated TG, reduced HDL cholesterol, and elevated FPG increased for groups with younger ages at first childbirth, but the percentages of only central obesity, elevated BP, and elevated TG significantly increased. Groups with younger ages at first childbirth also included more participants with a history of smoking (past and current), lower family income levels, lower education levels, hypertension, diabetes, breastfeeding, and oral contraceptives use; differences in these factors were significant. Alcohol consumption levels, regular walking, and history of dyslipidemia were not significantly different in the three groups. Table 2 presents the associations between age at first childbirth and central obesity using the logistic regression analysis with the backward elimination method. In model 1 (unadjusted), individuals with younger ages at first childbirth had higher ORs for central obesity than the other groups and had characteristics significantly associated with central obesity. However, in model 2 (adjusted for age), individuals with younger ages at first childbirth had attenuated ORs (with significance) for central obesity. In model 3 (lifestyle factors were added to model 2) and model 4 (socioeconomic factors were added to model 3), the attenuation of ORs (with significance) for central obesity was continued. In model 5 (reproductive factors were added to model 4), a younger age at first childbirth group was not significantly associated with central obesity (with significance) after full adjustment for confounding factors. In model 5 , age at first childbirth was excluded by the backward elimination method because $\mathrm{P}>0.10$ (Table 2). Table 3 (participants age, 64 years or less) and Table 4 (participants age, 65 years or more) present the associations between age at first childbirth and central obesity using the logistic regression analysis with the backward elimination method. In each table, age at first childbirth was excluded by the backward elimination method because $\mathrm{P}>0.10$ (Tables 3,4 ).

\section{DISCUSSION}

This study showed no significant association between age at first childbirth and central obesity after adjusting for confounding variables. Younger age at first childbirth was not a risk factor for central obesity for postmenopausal women. Our results were different from those of a

Table 2. Odds ratio for central obesity according to age at first childbirth, all participants

\begin{tabular}{lccc}
\hline & & & Age (y) \\
& Central obesity & $20-29$ & $\geq 30$ \\
\cline { 2 - 4 } Model 1 & $\leq 19$ & 1.00 (reference) & $0.712(0.516-0.984)^{\star}$ \\
Model 2 & $2.028(1.564-2.629)^{*}$ & 1.00 (reference) & $0.818(0.587-1.140)$ \\
Model 3 & $1.632(1.248-2.133)^{*}$ & 1.00 (reference) & $0.818(0.587-1.140)$ \\
Model 4 & $1.632(1.248-2.133)^{*}$ & 1.00 (reference) & $0.929(0.663-1.301)$ \\
Model 5 (before backward elimination) & $1.422(1.086-1.861)^{\star}$ & 1.00 (reference) & $0.991(0.701-1.401)$ \\
\hline
\end{tabular}

Values are presented as OR (95\% Cl). ORs and 95\% Cls were estimated by multiple logistic regression analyses. Model 1 (unadjusted): age at first childbirth; model 2 (adjusted): age was added to model 1; model 3 (adjusted): lifestyle factors (smoking, alcohol consumption, and regular walking) were added to model 2; model 4 (adjusted): socioeconomic factors (family income and education) were added to model 3; and model 5 (adjusted): reproductive factors (age at menarche, number of pregnancies, breastfeeding, oral contraceptive use, and reproductive period) were added to model 4 . In model 5 using backward elimination method, age at first childbirth was excluded from the table, because P-value $>0.10$. All continuous values with skewed distributions were log-transformed prior to the analysis.

$\mathrm{OR}$, odds ratio; $\mathrm{Cl}$, confidence interval.

${ }^{*} \mathrm{P}<0.05$.

Table 3. Odds ratio for central obesity according to age at first childbirth, participants aged $<65$ years

\begin{tabular}{lccc}
\hline & & \multicolumn{3}{c}{ Age (y) } & \\
\cline { 2 - 4 } Central obesity & $\leq 19$ & $20-29$ & $\geq 30$ \\
\hline Model 1 & $2.533(1.639-3.915)^{*}$ & 1.00 (reference) & $0.812(0.538-1.224)$ \\
Model 2 & $2.409(1.546-3.754)^{*}$ & 1.00 (reference) & $0.918(0.603-1.396)$ \\
Model 3 & $2.409(1.546-3.754)^{\star}$ & 1.00 (reference) & $0.918(0.603-1.396)$ \\
Model 4 & $1.795(1.140-2.825)^{*}$ & 1.00 (reference) & $0.973(0.663-1.494)$ \\
Model 5 (before backward elimination) & $1.621(1.011-2.600)^{*}$ & 1.00 (reference) & $1.076(0.692-1.673)$ \\
\hline
\end{tabular}

Values are presented as OR (95\% Cl). ORs and 95\% Cls were estimated by multiple logistic regression analyses. Model 1 (unadjusted): age at first childbirth; model 2 (adjusted): age was added to model 1; model 3 (adjusted): lifestyle factors (smoking, alcohol consumption, and regular walking) were added to model 2; model 4 (adjusted): socioeconomic factors (family income and education) were added to model 3; model 5 (adjusted): reproductive factors (age at menarche, number of pregnancies, breastfeeding, oral contraceptive use, and reproductive period) were added to model 4 . In model 5 using backward elimination method, age at first childbirth was excluded from the table, because $P$-value $>0.10$. All continuous values with skewed distributions were log-transformed prior to the analysis.

$\mathrm{OR}$, odds ratio; $\mathrm{Cl}$, confidence interval.

${ }^{*} \mathrm{P}<0.05$. 
Table 4. Odds ratio for central obesity according to age at first childbirth, participants aged $\geq 65$ years

\begin{tabular}{|c|c|c|c|}
\hline \multirow{2}{*}{ Central obesity } & \multicolumn{3}{|c|}{ Age (y) } \\
\hline & $\leq 19$ & $20-29$ & $\geq 30$ \\
\hline Model 1 & $1.423(1.026-1.973)^{\star}$ & 1.00 (reference) & $0.764(0.443-1.318)$ \\
\hline Model 2 & $1.374(0.988-1.910)$ & 1.00 (reference) & $0.748(0.433-1.293)$ \\
\hline Model 3 & $1.354(0.971-1.889)$ & 1.00 (reference) & $0.748(0.432-1.294)$ \\
\hline Model 4 (before backward elimination) & $1.268(0.906-1.775)$ & 1.00 (reference) & $0.831(0.476-1.450)$ \\
\hline Model 5 (before backward elimination) & $1.244(0.885-1.748)$ & 1.00 (reference) & $0.872(0.493-1.542)$ \\
\hline
\end{tabular}

Values are presented as OR (95\% CI). ORs and 95\% Cls were estimated by multiple logistic regression analyses. Model 1 (unadjusted): age at first childbirth; model 2 (adjusted): age was added to model 1; model 3 (adjusted): lifestyle factors (smoking, alcohol consumption, and regular walking) were added to model 2; model 4 (adjusted): socioeconomic factors (family income and education) were added to model 3; model 5 (adjusted): reproductive factors (age at menarche, number of pregnancies, breastfeeding, oral contraceptive use, and reproductive period) were added to model 4. In models 4 and 5 using backward elimination method, age at first childbirth was excluded from the table because P-value $>0.10$. All continuous values with skewed distributions were log-transformed prior to the analysis.

$\mathrm{OR}$, odds ratio; $\mathrm{Cl}$, confidence interval.

${ }^{*} \mathrm{P}<0.05$.

previous study ${ }^{17)}$ Previous analyses of the association between age at first childbirth and later health showed disadvantages for women. Childbirth at a young age and increased parity were independently associated with central obesity for women later in life. ${ }^{13,15,17)}$ Women with younger ages at first childbirth may have higher parity. ${ }^{18)}$ Multiparous women were at high risk for type 2 diabetes and cardiovascular disease later in life. ${ }^{19,20)}$ Several reasons might account for the causal relationship between multiparity, younger age at first childbirth, and central obesity. First, rapid fat accumulation and fat distribution changes occur during pregnancy. ${ }^{21)}$ Gestational weight gain may be the cause of obesity after childbirth and may increase the risk of obesity after menopause. Second, pregnancy at a younger age exposes immature reproductive organs to high doses of estrogen, which can have negative effects on the maternal organs. ${ }^{22,23)}$ Third, increased inflammatory cytokines due to pregnancy lead to insulin resistance, which may have deleterious effects on the normal development of maternal organs. ${ }^{1,24)}$

In the present study, we obtained data regarding the number of pregnancies instead of parity due to questionnaire limitations. We focused on the association between age at first childbirth and central obesity. To clarify the relationship between age at first childbirth and central obesity, various confounding factors were adjusted, such as age, lifestyle factors, socioeconomic factors, and reproductive factors. ${ }^{2,15,17)}$ The strength of the association between age at first childbirth and central obesity gradually decreased after adjustment. The association between age at first childbirth and central obesity was not significantly higher in model 5, which was adjusted for age, lifestyle factors, socioeconomic factors, and reproductive factors (Tables 2-4).

Apart from the physiological effects of pregnancy and childbirth, past experiences, accumulated stresses, and environmental conditions may have long-term effects on current health states. ${ }^{2)}$ In this study, the ages of participants varied from 44 to 80 years. Three groups that were separated according to age at first childbirth were a different birth cohort. Each group had different stress levels, experiences, and environmental conditions over the course of their lifetimes. For example, some experienced traumatic conditions such as the Korean War. Age was a strong confounding factor and important risk factor for cen- tral obesity. Therefore, to reduce the effects of age on the association between age at first childbirth and central obesity, participants were divided in to two age groups-those aged 64 years or less and 65 years or more-before the analysis (Tables 3, 4). Ultimately, this study found that a younger age at first childbirth is not a risk factor for central obesity in postmenopausal women.

The various conditions of our study participants were different from those of participants in a previous study. ${ }^{17)}$ The time interval from the previous study to the present study was almost 10 years. Different environmental conditions around the participants might have influenced the confounding factors. Data analyses of the present study were different from those of a previous study. In the previous study, alcohol intake was divided into two categories and exercise was considered instead of regular walking. Education and income levels were divided into five categories; reproductive factors included age at menopause, parity number, and hormone therapy, except for the reproductive period. Therefore, this may explain why the results of the previous study might be different from those of the present study.

The present study was a large, reliable, representative study of the Korean population using the most comprehensive statistical methods. Through this study, we were able to gain knowledge regarding the long-term effects of age at first childbirth on central obesity during the postmenopausal state. No association was found between age at first childbirth and central obesity in postmenopausal women.

Our study had several limitations. Given the cross-sectional design of the present study, the causal effects of age at first childbirth and other factors on central obesity later in life were not definite. The time interval from the age at first childbirth to central obesity at the time of the survey was too long; therefore, other unexpected factors might have influenced central obesity. Age at first childbirth, age at menopause, and age at menarche were based on self-reported questionnaires, which are subject to recall bias. In addition, we could not consider gestational medical problems such as gestational diabetes or gestational hypertension because of insufficient data. Our estimates of the reproductive period and postmenopausal period may have been inaccurate by up to 1 year because we only had information regarding the ages at 
menarche and menopause.

In conclusion, this study found no significant association between age at first childbirth and central obesity; however, our results might have been affected by the limitations of the available data. It might be possible to obtain different results without the study limitations, such as age adjustment, different birth cohorts, cross-sectional study type, recall bias, insufficient data, and sample size for missing data. Therefore, the results of this study should be interpreted after considering various effects before generalizing this information to other women. In addition, further prospective cohort studies should be conducted to examine the causal relationship between age at first childbirth and alterations of the waist circumference to identify the underlying physiological mechanisms in more detail.

\section{CONFLICT OF INTEREST}

No potential conflict of interest relevant to this article was reported.

\section{REFERENCES}

1. Kaaja RJ, Greer IA. Manifestations of chronic disease during pregnancy. JAMA 2005;294:2751-7.

2. Grundy E, Tomassini C. Fertility history and health in later life: a record linkage study in England and Wales. Soc Sci Med 2005;61:217-28.

3. Grundy E, Kravdal O. Fertility history and cause-specific mortality: a register-based analysis of complete cohorts of Norwegian women and men. Soc Sci Med 2010;70:1847-57.

4. Vandenheede H, Deboosere P, Gadeyne S, De Spiegelaere M. The associations between nationality, fertility history and diabetes-related mortality: a retrospective cohort study in the Brussels-Capital Region (2001-2005). J Public Health (Oxf) 2012;34:100-7.

5. Feng Y, Hong X, Wilker E, Li Z, Zhang W, Jin D, et al. Effects of age at menarche, reproductive years, and menopause on metabolic risk factors for cardiovascular diseases. Atherosclerosis 2008;196:590-7.

6. Lao XQ, Thomas GN, Jiang CQ, Zhang WS, Yin P, Schooling M, et al. Parity and the metabolic syndrome in older Chinese women: the Guangzhou Biobank Cohort Study. Clin Endocrinol (Oxf) 2006;65: 460-9.

7. Alberti KG, Zimmet P, Shaw J; IDF Epidemiology Task Force Consensus Group. The metabolic syndrome: a new worldwide definition. Lancet 2005;366:1059-62.

8. Lee SY, Park HS, Kim DJ, Han JH, Kim SM, Cho GJ, et al. Appropriate waist circumference cutoff points for central obesity in Korean adults. Diabetes Res Clin Pract 2007;75:72-80.

9. Mathew S, Kosmas CE, Siegel RR, Vittorio TJ. Toxicity of abdominal fat. Health 2013;5:96-9.

10. Toth MJ, Tchernof A, Sites CK, Poehlman ET. Effect of menopausal status on body composition and abdominal fat distribution. Int J Obes Relat Metab Disord 2000;24:226-31.
11. Park HS, Oh SW, Cho SI, Choi WH, Kim YS. The metabolic syndrome and associated lifestyle factors among South Korean adults. Int J Epidemiol 2004;33:328-36.

12. Ball K, Crawford D. Socioeconomic status and weight change in adults: a review. Soc Sci Med 2005;60:1987-2010.

13. Umberson D, Liu H, Mirowsky J, Reczek C. Parenthood and trajectories of change in body weight over the life course. Soc Sci Med 2011;73: 1323-31.

14. Alberti KG, Eckel RH, Grundy SM, Zimmet PZ, Cleeman JI, Donato KA, et al. Harmonizing the metabolic syndrome: a joint interim statement of the International Diabetes Federation Task Force on Epidemiology and Prevention; National Heart, Lung, and Blood Institute; American Heart Association; World Heart Federation; International Atherosclerosis Society; and International Association for the Study of Obesity. Circulation 2009;120:1640-5.

15. Patchen L, Leoutsakos JM, Astone NM. Early parturition: is young maternal age at first birth associated with obesity? J Pediatr Adolesc Gynecol 2017;30:553-9.

16. Laopaiboon M, Lumbiganon P, Intarut N, Mori R, Ganchimeg T, Vogel JP, et al. Advanced maternal age and pregnancy outcomes: a multicountry assessment. BJOG 2014;121 Suppl 1:49-56.

17. Cho GJ, Park HT, Shin JH, Kim T, Hur JY, Kim YT, et al. The relationship between reproductive factors and metabolic syndrome in Korean postmenopausal women: Korea National Health and Nutrition Survey 2005. Menopause 2009;16:998-1003.

18. Glasier A, Gulmezoglu AM, Schmid GP, Moreno CG, Van Look PF. Sexual and reproductive health: a matter of life and death. Lancet 2006;368:1595-607.

19. Fowler-Brown AG, de Boer IH, Catov JM, Carnethon MR, Kamineni A, Kuller LH, et al. Parity and the association with diabetes in older women. Diabetes Care 2010;33:1778-82.

20. Nicholson WK, Asao K, Brancati F, Coresh J, Pankow JS, Powe NR. Parity and risk of type 2 diabetes: the Atherosclerosis Risk in Communities Study. Diabetes Care 2006;29:2349-54.

21. Gunderson EP, Sternfeld B, Wellons MF, Whitmer RA, Chiang V, Quesenberry CP Jr, et al. Childbearing may increase visceral adipose tissue independent of overall increase in body fat. Obesity (Silver Spring) 2008;16:1078-84.

22. Pirkle CM, de Albuquerque Sousa AC, Alvarado B, Zunzunegui MV; IMIAS Research Group. Early maternal age at first birth is associated with chronic diseases and poor physical performance in older age: cross-sectional analysis from the International Mobility in Aging Study. BMC Public Health 2014;14:293.

23. Mauvais-Jarvis F, Clegg DJ, Hevener AL. The role of estrogens in control of energy balance and glucose homeostasis. Endocr Rev 2013;34: 309-38.

24. Chang CJ, Wu CH, Yao WJ, Yang YC, Wu JS, Lu FH. Relationships of age, menopause and central obesity on cardiovascular disease risk factors in Chinese women. Int J Obes Relat Metab Disord 2000;24: 1699-704. 